\title{
Chilean patients' perception of oral health-related quality of life after third molar surgery
}

This article was published in the following Dove Press journal:

Patient Preference and Adherence

6 September 2016

Number of times this article has been viewed

\author{
Pedro Christian Aravena ${ }^{1,2}$ \\ Felipe Delgado' \\ Hugo Olave' \\ Carolina Ulloa-Marin ${ }^{3}$ \\ Francisco Perez-Rojas ${ }^{4}$ \\ 'School of Dentistry, Faculty of \\ Medicine, ${ }^{2}$ Institute of Anatomy, \\ Histology and Pathology, Faculty \\ of Medicine, Universidad Austral \\ de Chile, Valdivia, ${ }^{3}$ Department of \\ Dentistry Based-Evidence, School \\ of Dentistry, Universidad de Chile, \\ Santiago, ${ }^{4}$ School of Medicine, \\ Universidad Autónoma de Chile, \\ Talca, Chile
}

Objective: To describe the perception of the quality of life in oral health based on the Health-Related Quality of Life instrument in its Spanish version (HRQOL-sp) in Chilean patients with third molar extraction surgery.

Patients and methods: A cross-sectional study. The HRQOL-sp was administered to dental patients at the Public Hospital in Rio Bueno in southern Chile treated for unilateral third molar extraction between March and June 2014. The instrument was applied by phone survey on the first, third, fifth, and seventh days after surgery. For the ordinal scale, the response was considered interference in the quality of life when patients selected the options "quite a bit of trouble" or "lots of trouble" for oral function and general activity; and selected complications-related signs and symptoms, a pain level score with a Verbal Rating Scale (range 0 to 7), and worst pain perceived. The patient's sociodemographic data, type of surgery, and the quality of life level were analyzed according to the domains of the HRQOL-sp instrument.

Results: A total of 106 patients were selected (age: $20.4 \pm 7.39$ years; $71.7 \%$ women) and a total of 127 extracted third molars. On the first day of follow-up, most patients reported interference in their quality of life. The main problems were difficulty opening the mouth $(50.94 \%)$ and swelling (83.02\%). The worst symptom perceived was "bad breath" $(>31 \%)$ and the worst pain felt was a mean of $4.31 \pm 1.62$ on the Verbal Rating Scale. All items gradually reduced until the seventh day.

Conclusion: The HRQOL-sp revealed substantial interference in the quality of life on the first postoperative day. It is suggested that the risk factors associated with quality of life be analyzed and the instrument in Spanish be validated.

Keywords: quality of life, complications, extraction, third molar, dentistry, oral surgery

\section{Introduction}

Third molar extraction is one of the most frequent surgical procedures performed in oral surgery. ${ }^{1}$ Postoperative complications associated with this procedure include pain, swelling, and sensitivity, which alter the normal pattern of people's daily activities, thus affecting their quality of life $^{2}$ essentially for the first three postoperative days, but these almost completely disappear by the fifth day. ${ }^{3,4}$

Health-related quality of life (HRQOL) is considered to be a subset of the quality of life that assesses symptoms, psychological aspects, and function. In turn, quality of life in oral health reflects a person's comfort when eating, sleeping, and during social interaction, as well as their self-esteem and satisfaction with their oral health. ${ }^{5}$ Shugars et $\mathrm{al}^{6}$ designed an instrument to measure HRQOL after third molar surgery that was modified by Conrad et al. ${ }^{7}$ This instrument is used specifically to measure patients' perception after surgery in four domains (oral function, general activity, signs and symptoms, and pain). 
In the Hispanic population, the measurement of quality of life after a third molar extraction surgery has been studied ${ }^{3,8,9}$ noting a reduction in quality of life during the first five postoperative days with the presence of pain and an alteration in their working life. However, the HRQOL instrument ${ }^{6,7}$ used on the Hispanic population does not exist in the electronic databases; therefore, this report would contribute to the recognition of the different domains that influence recovery and quality of life in patients after a third molar extraction.

The objective of this study is to describe the use and perception of quality of life in oral health based on the HRQOL instrument in its Spanish version in patients who underwent third molar extraction surgeries in the Chilean population.

\section{Patients and methods}

A cross-sectional study was designed to assess dental patients in the Public Hospital in Rio Bueno in Southern Chile treated for a third molar extraction in March and June 2014. The selection criteria were patients over the age of 14 years, and American Society of Anesthesiologists who signed the informed consent agreeing to participate in the study. Psychiatric patients, pregnant or breastfeeding women, patients with underlying chronic pathologies that require them to consume antibiotics, nonsteroidal anti-inflammatory drugs or corticosteroids, and those who did not complete the protocol and time required by the study were excluded. The study was approved by the Ethics and Scientific Committee of the Health Service of Valdiva (no 041/2014) and all patients signed written informed consent.

The number of patients to analyze is based on the recommendations of Streiner and Norman ${ }^{10}$ by means of the analysis of a minimum of five patients per item of the scale applied. Considering a 95\% confidence interval, 5\% error margin, and $50 \%$ heterogeneity, the evaluation of a minimum of 77 patients selected by a nonprobabilistic sampling method was needed.

\section{Adaptation of the HRQOL scale to a Spanish version}

Two researchers (HO, FD) translated the original HRQOL instrument $^{6,7}$ into Spanish and this was revised by an external native English-speaking researcher. A focus group with three surgeons and 15 patients from the Hospital's dental service was used for the face review and construct validity assessment. In turn, the alternatives on the Likert scale were reduced from five to only four, as the presence of an odd alternative number in the original scale ("some trouble") directed the responses to an intermediate value. ${ }^{11}$ In order to quantify the signs and symptoms, the five alternatives proposed by Shugars et $\mathrm{al}^{6}$ were modified for dichotomous values (present/absent), where the alternatives "quite a bit of trouble" and "lots of trouble" on the original scale were taken as "present" according to the definition suggested by White et al. ${ }^{4}$ Then, a pilot study was carried out by applying the scale to 21 patients treated with a third molar extraction surgery, using the adapted scale on two occasions on the first day of postoperative follow-up. The internal consistency was analyzed with the test-retest reliability of the scale, obtaining an interclass correlation above $80 \%$ in all items (16). Finally, a document in Spanish (HRQOL-sp) was obtained from the original HRQOL instrument. ${ }^{6,7}$

The HRQOL-sp instrument has four domains: oral function (eating, chewing, opening one's mouth, and being able to talk), general activity (sleep, daily routine, social life, and hobbies or sports), signs or symptoms associated with postoperative complications, and level of pain measured by Verbal Rating Scale (VRS). For oral function and general activity, four alternatives were presented on a Likert scale: "no trouble", "little trouble", "quite a bit of trouble", and "lots of trouble". The presence of signs and symptoms was valued dichotomously (yes/no) and the pain level and worst pain felt during the follow-up day was measured with a VRS from 1 (without pain) to 7 points (worst pain imaginable).

\section{Third molar extraction surgery}

A maxillofacial surgeon (CU) with more than 15 years of experience performed the third molar extraction unilaterally. A researcher (FD) registered the participant's name, age, sex, contact phone, and address. All patients were administered antibiotic prophylaxis of $2 \mathrm{~g}$ of amoxicillin orally 1 hour before surgery or $600 \mathrm{mg}$ of clindamycin 1 hour before surgery for patients who were allergic to penicillin byproducts. Two cartridges of $1.8 \mathrm{~mL} 2 \%$ lidocaine 1:80,000 epinephrine (Xylonor 2\% Especial, Septodont ${ }^{\circledR}$ ) were used as the inferior alveolar nerve, lingual nerve, and buccal nerve block anesthesia. A sterile surgical field was prepared, disinfecting the skin with gauze moisturized with $2 \%$ chlorhexidine. The surgical technique consisted of a mucoperiosteal flap, osteotomy, and dental section with a round carbide bur using a $0.9 \%$ saline solution (Apiroflex ${ }^{\circledR}$ ) as the refrigerant and a medium straight elevator for the subsequent tooth dislocation and removal. The pericoronary sack was eliminated with a bone curette and the wound was irrigated with $0.9 \%$ saline solution. All patients were indicated a relative rest for 3 days, cold diet for 24 hours, and a soft diet for 3 days. They were all prescribed $1 \mathrm{~g}$ amoxicillin every 12 hours for 7 days after 
surgery, $50 \mathrm{mg}$ diclofenac sodium every 8 hours for 3 days, and $500 \mathrm{mg}$ acetaminophen every 8 hours for 3 days.

\section{Application of the scale}

Before the surgery, a researcher (HO) introduced the scale and gave a copy to each patient together with a verbal explanation of its content and how to answer it. To collect the answers, phone calls were made on the first, third, fifth, and seventh days after surgery, following the recommendation of Susarla et $\mathrm{al}^{12}$ as a postoperative follow-up method. During this call, the researcher read the questions, giving the patient the options for the response in the domain of oral function and general activity, the presence of signs or symptoms, and the quantification of pain. If the patient did not answer the phone calls during the monitoring or if he/she answered incompletely, his/her data and records were discarded from the study.

On the seventh day after surgery, the patients returned to the Dental Service for a check-up. Finally, the data were stored in a Google Drive electronic database (Google Inc. Mountain View, CA, USA), encoding the patients' registration number and personal data.

\section{Data analysis}

Variables considered as risk factors associated with postoperative complications in third molar extraction surgery were recorded for each patient: age, sex (male/female), schooling (primary/secondary/higher education), residence (urban/rural), and tobacco consumption. The reason for the surgery, number of teeth extracted, and time of surgery (less or more than 20 minutes) $)^{13}$ were recorded as well as the difficulty level based on the position of the third molar with higher surgical complexity according to the Pederson scale (slightly, moderately, or very difficult). ${ }^{14,15}$ It was considered as a substantial interference in quality of life if the patients marked the responses "lots of trouble" or "quite a bit of trouble" in each of the measured items in the domains of oral function and general activity on the scale; the presence of signs or symptoms (yes/no), level of pain with a VRS scale (range 1 to 7 points), and presence of the worst pain felt were defined when the patient perceived more than 5 points on the VRS scale.

Descriptive statistics calculated the mean and standard deviation for the continuous variables and the use of frequencies and distribution percentage were used for the categorical variables. Analytical statistics was conducted to determine an item of HRQOL-sp scale and postoperative signs and symptoms were a difference of statistical significance between postoperative days (chi-squared; $P<0.05$ ). The results were presented as mean on tables and figures using the SPSS Software 20.0 (IBM Corporation, Armonk, NY, USA).

\section{Results}

A total of 110 patients participated in the study. Four did not answer the phone calls, and therefore only 106 were selected (age: $20.78 \pm 7.1$ years; $71.7 \%$ female). The removal of third molars was performed in 50 patients and in 56 patients removal of third molars upper and lower was simultaneously performed. In $45.28 \%$ of the cases, the extraction was performed as prophylaxis. The most frequently extracted tooth was \#17 (49.06\%) followed by \#32 (46.2\%). In all, $71.76 \%$ of the surgeries lasted between 20 and 40 minutes, considering that $60.38 \%$ of the patients had a moderately difficult surgery (22) (Table 1).

All the items that integrate the oral function and general activity concepts from the HRQOL-sp presented a higher frequency of "quite a bit of trouble" and "lots of trouble" as responses on the first postoperative day with a gradual decrease until the end of the follow-up; the exception was the item "sleep", which peaked on the third postoperative day $(P<0.05)$. The patients' detailed responses in relation to oral function and general activity are presented in Table 2 .

In relation to the signs and symptoms, on the first day there were a higher number of patients with cheek swelling (83.02\%) and bleeding (33.96\%). The least perceived symptom during the follow-up week was nausea. On the third day,

Table I Sociodemographic characteristics and clinical data obtained after the surgery of patients included in the study $(n=106)$

\begin{tabular}{lll}
\hline Variable & Item & $\mathbf{( \% )}$ \\
\hline Sex & Women & 71.7 \\
Residence & Urban & 80.02 \\
& Rural & 19.98 \\
Educational level & Primary & 28.3 \\
& Secondary & 54.72 \\
& University & 16.98 \\
Tobacco (smoker) & No & 75.47 \\
& $<5$ cigarettes per day & 18.87 \\
Extraction motive & Prophylaxis & 45.28 \\
& Orthodontia & 34.91 \\
& Prior history of pericoronitis & 13.21 \\
& Other (auto-report of pain) & 3.77 \\
& Second molar compromise & 1.89 \\
Number of third molar & Prosthesis & 0.94 \\
extracted by patient & Lower third molar & 47.17 \\
Time length of surgery & Upper and lower third molar & 52.83 \\
& $<20$ minutes & 28.3 \\
Surgery difficulty & Between 20 and 40 minutes & 71.7 \\
& Slightly difficult & 18.87 \\
& Moderately difficult & 60.38 \\
& Very difficult & 20.75 \\
\hline
\end{tabular}


Table 2 Percentage of patients who expressed a substantial interference in their daily activities according to the responses "quite a bit of trouble" and "lots of trouble" for oral function and general activity $(n=106)$

\begin{tabular}{|c|c|c|c|c|c|c|}
\hline Domain & Item & Day I & Day 3 & Day 5 & Day 7 & $P$-value* \\
\hline \multirow[t]{4}{*}{ Oral function } & Eating the food that you want & 32 & 28 & 9 & 2 & $<0.001$ \\
\hline & Chewing & 48 & 38 & 24 & 10 & $<0.001$ \\
\hline & Open mouth to the maximum & 51 & 45 & 17 & 9 & $<0.001$ \\
\hline & Talking & 9 & 8 & 3 & 2 & 0.68 \\
\hline \multirow[t]{4}{*}{ General activity } & Sleeping & 12 & 23 & 7 & 3 & $<0.001$ \\
\hline & Continue with daily routine & 19 & 16 & 4 & 3 & 0.001 \\
\hline & Continue with social life & 18 & 15 & 3 & 2 & $<0.001$ \\
\hline & Playing sports or hobbies & 47 & 42 & 27 & 8 & $<0.001$ \\
\hline
\end{tabular}

Note: *Chi-square (level of significance $P=0.05$ ).

the most frequent symptoms were bad taste or bad breath (50.94\%) and the presence of bleeding $(33.96 \%)(P<0.05)$. All signs and symptoms subsided day by day. Details of the symptomatology recorded are shown in Table 3.

The average pain perceived on the first day was 3.09 \pm 1.15 points on the VRS scale. Similarly, the response percentage of the worst pain felt considered as pain that interferes in quality of life was most frequent in patients on the third postoperative day (11.32\%) (Figure 1).

\section{Discussion}

The perception of quality of life in oral health based on the HRQOL-sp instrument in patients who underwent third molar extraction surgeries in the Chilean population was mainly affected in the first 3 days after surgery. Cheek swelling and bad taste or bad breath were the most frequent symptoms for patients during the postoperative days. Pain levels gradually subsided with time.

The HRQOL instrument was adapted in our study into Spanish to be applied to the Chilean population. To achieve this, a quantitative methodology was used by means of sight translation into Spanish, a review of the scale in expert meetings, and its pilot use with patients to validate its content. ${ }^{16}$ In turn, we considered reducing the Likert scale response range from five alternatives to only four, as the presence of an

Table 3 Percentage of patients with signs or symptoms $(n=106)$

\begin{tabular}{llllll}
\hline Signs and symptoms & Day I & Day 3 & Day 5 & Day 7 & P-value* \\
\hline Cheek swelling & 83 & 76 & 56 & 25 & $<0.001$ \\
Hematomas & 17 & 22 & 16 & 10 & 0.14 \\
Postsurgical bleeding & 34 & 34 & 25 & 19 & 0.04 \\
Nausea & 9 & 8 & 5 & 2 & 0.14 \\
Bad taste or bad breath & 47 & 51 & 46 & 31 & 0.02 \\
Food accumulation in the & 13 & 24 & 23 & 21 & 0.2 \\
place of the extraction & & & & & \\
\hline
\end{tabular}

Note: *Chi-square (level of significance $P=0.05$ ). odd alternative on the original scale ("some trouble") 6 could mean the responses have an intermediate value, allowing respondents to avoid giving a concrete answer. ${ }^{11}$

For the items average age, sex (frequently females), education level of the study patients, and tobacco consumption, the main reason for third molar extraction (prophylactic indication) and length of surgery (between 20 and 40 minutes) coincide with the use of this scale in previously reported studies. ${ }^{4,7}, 17$ In terms of oral function, our results vary from previous studies. On the first day after surgery, our study shows less substantial interference in patients' quality of life, approaching as a maximum half the patients with trouble chewing or opening their mouth; these results differ from the almost three-quarters seen in the other studies where patients were involved on extraction of all four third molars under intravenous anesthesia ${ }^{4,7,17}$ (Table 4). Despite this difference in signs and symptoms, the items bleeding, accumulation of food, and bad taste or bad breath are quite similar to those previously found. However, most of our patients (more than $80 \%$ ) reported no major interference when returning to their daily routines in the days after surgery; this is why the high frequency of swelling would not be an impediment to patients' quality of life after surgery.

Our results show that the quality of life of the patients was mainly affected on the first and third days after surgery. However, if we analyze the variables of the scale with previous studies, $4,7,17$ we observe fewer responses of "lots of trouble" and "quite a bit of trouble" on the first follow-up day after surgery with the exception of swelling, hematoma, and bad taste or bad breath compared to our sample (Table 4).

Regarding perceived pain, our results reported a lower perception of pain than previous studies. ${ }^{4,7,17}$ Nevertheless, the worst pain felt was similar on the first day after surgery to what was reported by White et al, ${ }^{4}$ although the similarity changes on the fifth day, when our patients' perception of 
Table 4 Sociodemographic variables and percentage of responses "lots of trouble" and "quite a bit of trouble" used on the HRQOL scale on the first day after surgery in previous studies

\begin{tabular}{|c|c|c|c|c|c|}
\hline Variables & Items & $\begin{array}{l}\text { Conrad et } \text { al }^{7} \\
\text { n: } 201\end{array}$ & $\begin{array}{l}\text { White et } \mathrm{al}^{4} \\
\mathrm{n}: 630\end{array}$ & $\begin{array}{l}\text { Shugars et } \mathrm{al}^{17} \\
\mathrm{n}: 63\end{array}$ & $\begin{array}{l}\text { Aravena et al } \\
\text { n: } 106\end{array}$ \\
\hline \multirow[t]{5}{*}{ Sociodemographic } & Women & 67 & 61 & 60 & 72 \\
\hline & Average age (years) & 23 & 21 & 21 & 20 \\
\hline & Over 25 years & 26 & - & 15 & 19 \\
\hline & $\begin{array}{l}\text { Completed educational } \\
\text { level over secondary school }\end{array}$ & - & 87 & 52 & 72 \\
\hline & Tobacco consumption & - & 18 & - & 25 \\
\hline \multirow[t]{4}{*}{ Oral function } & Eating & - & 68 & 71 & 32 \\
\hline & Chewing & 85 & 78 & 75 & 48 \\
\hline & Open the mouth & 78.5 & 71 & 7I & 51 \\
\hline & Talk & 37.5 & 23 & 19 & 9 \\
\hline \multirow[t]{4}{*}{ General activity } & Sleep & 24 & 19 & 14 & 12 \\
\hline & Daily routine & 60 & 46 & 41 & 19 \\
\hline & Social life & 61.5 & 51 & 58 & 18 \\
\hline & Recreation & 70 & 63 & 59 & 47 \\
\hline \multirow[t]{6}{*}{ Signs and symptoms } & Swelling & $6 I *$ & $46 *$ & 46 & 83 \\
\hline & Hematomas & - & 5 & - & 17 \\
\hline & Bleeding & 48 & 34 & - & 34 \\
\hline & Nausea & 24 & 18 & 22 & 9 \\
\hline & Bad taste/bad breath & 48 & 35 & - & 47 \\
\hline & Food accumulation** & 13 & 14 & - & 24 \\
\hline \multirow[t]{2}{*}{ Pain } & Average & 29 & 20 & 13 & 7.5 \\
\hline & Worst & 63.5 & 54 & 48 & 46 \\
\hline
\end{tabular}

Notes: *Day 2 after the surgery, **Day 3 after the surgery. Bold value: Authors with highest percentage compared between studies.

Abbreviation: HRQOL, health-related quality of life.

pain subsided considerably (10.38\%) compared to what was found by White et $\mathrm{al}^{4}(22 \%)$ on the same day.

In general, our results show a lower frequency of problems registered by the HRQOL scale that are harmful to quality of life from the first follow-up day after the surgery. This variation could be the result of the surgical conditions used, such as unilateral third molar extraction, the location and difficulty of the surgery, the use of antibiotic prophylaxis, or the postoperative medication of antibiotics and nonsteroidal anti-inflammatory drugs. Similar reports of

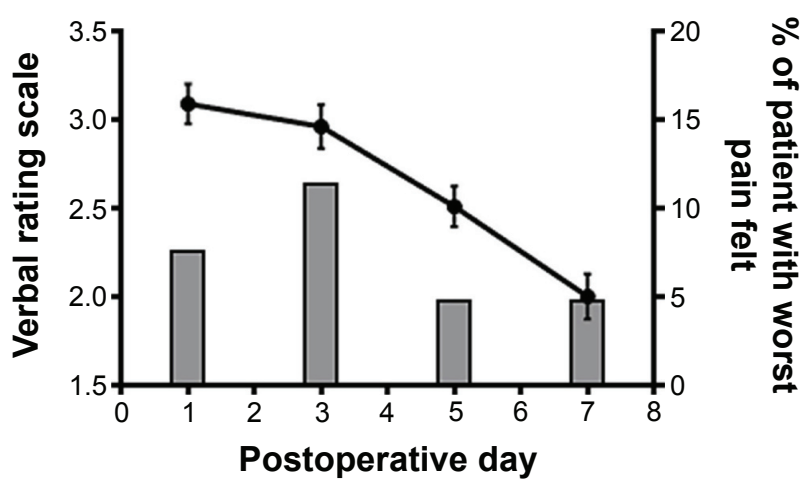

Figure I Level of pain perceived according to the verbal Rating Scale (VRS). Notes: Left: mean of points and standard deviation of VRS. Right: Percentage of patients with worst pain felt by postoperative day. the use of the HRQOL scale have reported on the extraction of all four third molars in one surgery, ${ }^{4,7}$ finding that the position of third molars is associated with technical difficulties in their extraction and that this directly influences lower quality of life-related oral health. ${ }^{18}$ These conclusions agree with a moderate surgical complication level according to the Pederson scale ${ }^{14,15}$ observed in our study, which could have favored a lower perception of signs and symptoms associated with the surgery and, with that, a better quality of life. ${ }^{19}$

The use of antibiotics and anti-inflammatories could also have affected the decrease in postoperative problems in our study. Although to date there has been no consensus about the effectiveness of postoperative antibiotic therapy in the control of complications in third molar surgery, ${ }^{20}$ the use of amoxicillin prophylactically and postoperatively is effective in the prevention of postoperative complications compared to the use of a placebo. ${ }^{21}$

Data collection from the HRQOL scale through phone calls could have contributed to patient recovery. Susarla et $\mathrm{al}^{12}$ demonstrated that patients who received phone calls to check on their condition after a tooth extraction were more satisfied (more than 90\%) and did not need a postoperative clinic visit. In order to reduce the need for a second visit to the Dental service at the Public Hospital in Rio Bueno, the 
researchers adopted this control and register protocol of the study variables, data that show a lower frequency of problems that could affect patients' quality of life compared to preliminary reports.

The limitations of our study are related to the use of a self-report, anatomical variability, patients' sex and age, and lack of control of drug use, which could lead to bias in the results. In the adaptation of the scale into Spanish, an interview strategy and a pilot group were used to establish the face validity of the instrument. However, it is necessary to design a study of validity and reliability of the instrument by comparing the results of the scale with an instrument that describes the level of postoperative complications in third molar extractions ${ }^{22}$ and analyze the internal consistency of the items. ${ }^{10}$ The delivery of the survey on paper and the guided reading through phone could have generated a bias in the results by not visually verifying the responses mentioned to the patients, as respondents who use scales tend to overestimate their state of health.

In conclusion, the HRQOL instrument adapted into Spanish found a substantial interference in the quality of life, mainly on the first day after surgery. However, the results were better than the use of this scale in previous studies, mainly because of the patients' factors, number of extracted teeth, the antibiotic and analgesic protocols, and the control and use of the scale through phone calls. It is suggested that the HRQOL instrument into Spanish be validated through psychometric studies in health and the protocols of patient care and control after a third molar extraction surgery standardized to improve the quality of life index.

\section{Disclosure}

The authors report no conflicts of interest in this work.

\section{References}

1. Chaparro-Avendaño A, Pérez-García S, Valmaseda-Castellón E, BeriniAytés L, Gay-Escoda C. Morbidity of third molar extraction in patients between 12 and 18 years of age. Med Oral Patol Oral Cir Bucal. 2005; 10(5):422-431.

2. Susarla SM, Dodson TB. Risk factors for third molar extraction difficulty. J Oral Maxillofac Surg. 2004;62(11):1363-1371.

3. Colorado-Bonnin M, Valmaseda-Castellón E, Berini-Aytés L, GayEscoda C. Quality of life following lower third molar removal. Int $J$ Oral Maxillofac Surg. 2006;35(4):343-347.
4. White RP Jr, Shugars DA, Shafer D, Laskin D, Buckley M, Phillips C. Recovery after third molar surgery: clinical and health-related quality of life outcomes. J Oral Maxillofac Surg. 2003;61:535-544.

5. Sischo L, Broder HL. Oral health-related quality of life: what, why, how and future implications. J Dent Res. 2011;90(11):1264-1270.

6. Shugars DA, Benson K, White RP Jr, Simpson KN, Bader JD. Developing a measure of patient perceptions of short-term outcomes of third molar surgery. J Oral Maxillofac Surg. 1996;54(12):1402-1408.

7. Conrad SM, Blakey GH, Shugars DA, Marciani RD, Phillips C, White RP Jr. Patients' perception of recovery after third molar surgery. J Oral Maxillofac Surg. 1999;57(11):1288-1294.

8. Limeres J, Sanromán JF, Tomás I, Diz P. Patients' perception of recovery after third molar surgery following postoperative treatment with moxifloxacin versus amoxicillin and clavulanic acid: a randomized, double-blind, controlled study. J Oral Maxillofac Surg. 2009;67(2): 286-291.

9. Sancho-Puchades M, Valmaseda-Castellón E, Berini-Aytés L, Gay-Escoda C. Quality of life following third molar removal under conscious sedation. Med Oral Patol Oral Cir Bucal. 2012;17(6):994-999.

10. Streiner DL, Norman GR. Health Measurement Scales: a Practical Guide to Their Development and Use. New York: Oxford University Press; 2008 .

11. Hernandez A, Espejo B, González-Romá V. The functioning of central categories middle level and sometimes in graded response scales: does the label matter. Psicothema. 2006;18(2):300-306.

12. Susarla SM, Black R, Dodson TB. After dentoalveolar surgery, most patients are satisfied with telephone follow-up. J Oral Maxillofac Surg. 2011;69(8):2099-2105.

13. Benediktsdóttir IS, Wenzel A, Petersen JK, Hintze H. Mandibular third molar removal: risk indicators for extended operation time, postoperative pain, and complications. Oral Surg Oral Med Oral Pathol Oral Radiol Endod. 2004;97(4):438-446.

14. Pederson GW. Oral Surgery. Philadelphia: WB Saunders; 1988.

15. Koerner KR. The removal of impacted third molars principles and procedures. Dent Clin North. 38(2):255-278.

16. Pesudovs K, Burr J, Harley C, Elliott D. The development, assessment, and selection of questionnaires. Optom Vis Sci. 2007;84(8):663-674.

17. Shugars DA, Gentile MA, Ahmad N, et al. Assessment of oral healthrelated quality of life before and after third molar surgery. $J$ Oral Maxillofac Surg. 2006;64(12):1721-1730.

18. Negreiros RM, Biazevic MG, Jorge WA, Michel-Crosato E. Relationship between oral health-related quality of life and the position of the lower third molar: postoperative follow-up. J Oral Maxillofac Surg. 2012; 70(4):779-786.

19. Yuasa H, Kawai T, Sugiura M. Classification of surgical difficulty inextracting impacted third molars. Br J Oral Maxillofac Surg. 2002;40(1): $26-31$.

20. Omens MA, Forouzanfar T. Antibiotic prophylaxis in third molar surgery: a review. Oral Surg Oral Med Oral Pathol Oral Radiol. 2012; 114(6):5-12.

21. López-Cedrún JL, Pijoan JI, Fernández S, Santamaria J, Hernández G. Efficacy of amoxicillin treatment in preventing postoperative complications in patients undergoing third molar surgery: a prospective, randomized, double-blind controlled study. J Oral Maxillofac Surg. 2011; 69(6):5-14.

22. Aravena PC, Astudillo P, Manterola C. Design of a scale for measuring post-surgical complications in third molar surgery. Int J Oral Maxillofac Surg. 2014;43(8):1008-1014. 
Patient Preference and Adherence

Dovepress

\section{Publish your work in this journal}

Patient Preference and Adherence is an international, peer-reviewed, open access journal that focuses on the growing importance of patient preference and adherence throughout the therapeutic continuum. Patient satisfaction, acceptability, quality of life, compliance, persistence and their role in developing new therapeutic modalities and compounds to optimize

Submit your manuscript here: http://www.dovepress.com/patient-preference-and-adherence-journ clinical outcomes for existing disease states are major areas of interest for the journal. This journal has been accepted for indexing on PubMed Central The manuscript management system is completely online and includes a very quick and fair peer-review system, which is all easy to use. Visit http://www. dovepress.com/testimonials.php to read real quotes from published authors. 